\title{
EDITORIAL
}

\section{Real estate of monolayer permeability: location location location}

\author{
Corey Hardin ${ }^{1}$, Jeffrey J Fredberg ${ }^{2}$ and Ramaswamy Krishnan ${ }^{3}$
}

Laboratory Investigation (2013) 93, 148-150; doi:10.1038/labinvest.2012.168

${ }^{1}$ Division of Pulmonary and Critical Care Medicine,

Massachusetts General Hospital, Boston, MA USA; ${ }^{2}$ Harvard School of Public Health, Boston, MA, USA and ${ }^{3}$ Center for Vascular Biology, Beth Israel Deaconess Medical Center Boston, MA, USA Correspondence: E-mail: jjf@harvard.edu
| $\mathrm{n}$ understanding real estate, as we all know, the preeminent notion is location. Location is foremost mainly because real estate can be so heterogeneous spatially. From one city to the next or from one neighborhood to the next, as well as from one street to the next or even from one house to the next, the desirability and the price of real estate can vary rather dramatically. Broad-brush generalizations such as average house values might be useful and remain valid while missing altogether a degree of heterogeneity and particularity that can belie any average. For example, who among us would buy a house-sight unseen-based only upon the neighborhood? If it is to be your house, the details matter. The immediate neighbors matter.

Just so, in understanding permeability of the endothelial or epithelial monolayer, we have suspected for some time that paracellular gaps might be distributed in a fashion that is spatially heterogeneous, ${ }^{1}$ and that details matter. For a homogeneous cell population and a given measure of average monolayer permeability, not all gaps are necessarily created equal. Indeed, one wonders if a small fraction of gaps might account for most of the leak. And what is the scale of the gaps? Do gaps arise in clusters in a cooperative fashion or on the contrary, do they inhibit one another? Regarding the characterization of the morphology and physiology of these paracellular gaps, however, our abilities in the laboratory have been more aspirational than real, and as such we have settled for average measures that necessarily blur the details over rather large scales of space and time. For example, progressive increases in lung weight or in wet weight/dry weight ratio provide unequivocal evidence of pulmonary vascular leak, but these are organ-scale metrics, and slow ones at that. ${ }^{2}$ Changes of electrical resistance are tremendously faster and somewhat more localized, as are measurements of fluxes of certain molecular tracers. ${ }^{3}$ However to resolve and quantify monolayer permeability at a subcellular scale comparable to the dimensions of the gap itself, no methods have been available and, as such, we have been navigating largely in the dark.

What is needed, of course, is an assay that is high-resolution. Being fast and high throughput would be a bonus. Using such an assay, we could turn on the lights, as it were, and thus visualize for the first time, the gaps between living cells even if only in a model culture system. In this issue, Dubrovskyi et $a l^{4}$ describe an elegantly simple technical approach that does just that, and opens the door to high throughput measurements as well. In brief, they have grown endothelial cell monolayers on glass coverslips coated with biotinlabeled substrates (collagen or gelatin). When they subsequently treat the monolayers with FITClabeled avidin, the cellular layer acts as a mask that permits FITC-avidin binding only in those areas where the substrate is exposed by the formation of a paracellular gap. The resulting fluorescent signal correlates well with events at the cell junction as revealed by actin and VE-cadherin staining. Far more importantly, the fluorescent image comprises a detailed map of local paracellular gaps. Using this approach, paracellular gap formation is visualized and its heterogeneity quantified on the subcellular scale. However, if gap formation is spatially heterogeneous, the next logical questions are, 'Why do gaps form?', 'Why are the gaps so heterogeneous?', 'How does local cytoskeletal remodeling relate to local leakiness?' and, ultimately, 'What is the basic mechanism of gap formation?'

Empirically, we know that certain agonists like thrombin promote endothelial gap formation, ${ }^{3}$ as do stretch events associated with mechanical 

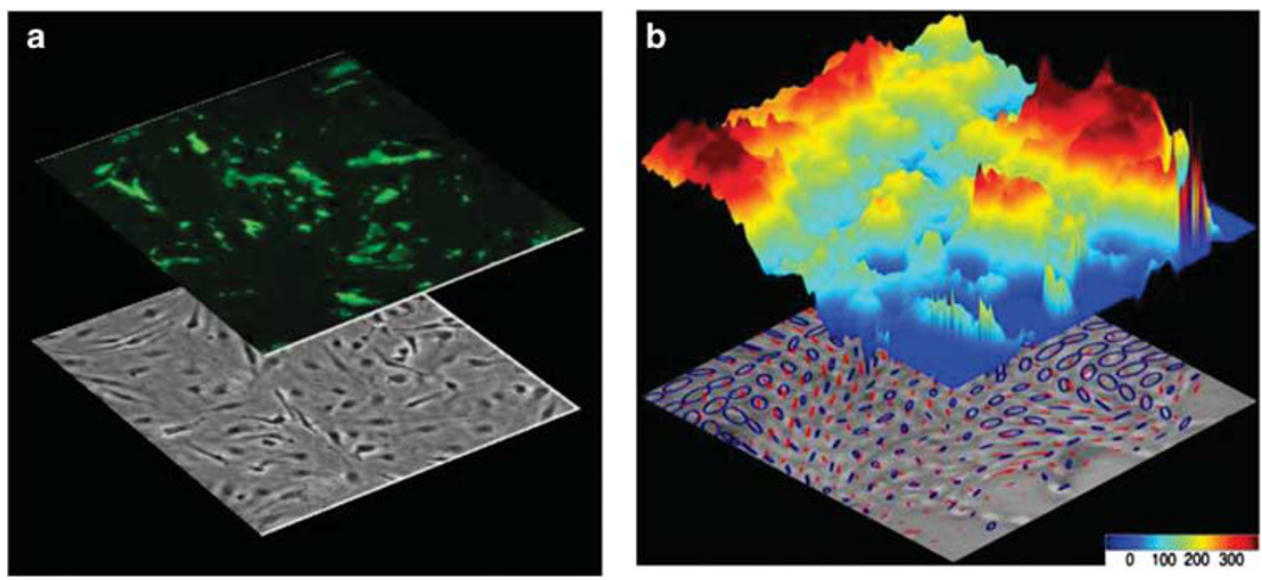

Figure 1 Heterogenity of forces and gaps. In panel (a), the phase contrast image of a cellular monolayer appears relatively homogeneous, yet overlay of the gap image reveals great spatial heterogeneity in the degree of gap formation. Panel (b) depicts a similar monolayer with an overlay of intercellular forces. ${ }^{12}$ The striking force heterogeneity is known to arise from the highly collective nature of intercellular stress transmission, but does this also explain the heterogeneity exhibited in panel (a).

ventilation, ${ }^{5,6}$ actomyosin contractility ${ }^{7}$ and stiffening of the cellular substrate. ${ }^{8}$ Taken together, such empirical observations lead to the reasonable suspicion that the mechanism of gap formation involves physical forces. ${ }^{9}$ It is reasonable to imagine, further, that if a gap forms between adjacent cells of the monolayer, then unseen but highly localized physical forces must be at work within each cell either to create each gap or to respond to its formation. These forces might include transmission of adhesive forces between the cell base and the cell substrate across focal adhesions and integrins, transmission of adhesive forces between the cell and its immediate neighbors, as through adherens junctions or tight junctions, and transmission of contractile forces along stress fibers. ${ }^{10}$ The notion, then, is that intercellular junctions are maintained by some balance of contractile forces and adhesive forces, and that gap formation arises when contractile forces become too large or adhesive forces become too small. ${ }^{9}$ However, if an imbalance between contractile versus adhesive forces might explain why gaps might form, such an imbalance fails to explain why gap formation is innately heterogeneous, as revealed in the current study.

Like the gaps themselves, the physical forces expressed across the cell-cell junction had been virtually invisible. However, with the advance of Dubrovskyi et al. and others, ${ }^{11-13}$ both the gaps and the intercellular force landscape that creates them are visible. Rather than being smooth and homogeneous, the intercellular force landscape is rugged and heterogeneous. Force heterogeneity arises from the collective and cooperative action amongst clusters of roughly 10-30 cells. ${ }^{12-15}$ Can such a physical picture explain the heterogeneity of gap formation? That is to say, can the peaks of the intercellular force landscape define the vulnerable loci of gaps with agonist challenge or stretch? If true, this would imply that measurements of central tendency, whether mean or median, no matter how detailed, would not suffice to explain the large-scale cooperative physical events that induce gaps. Moreover, at any particular instant, gaps would not be tied to any particular position or any particular cell. It would be a consequence of a global 'tug-of-war' amongst clusters of cells (Figure 1).

To rigorously test this picture, it remains to be seen whether the approach of Dubrovskyi et al. can be combined with the simultaneous assessment of intercellular force in the same preparation. Nevertheless, this work makes amenable a radical new frame of thought and with it, an unanticipated mechanistic perspective.

\section{DISCLOSURE/CONFLICT OF INTEREST}

The authors declare no conflict of interest.

1. McVerry BJ, Peng X, Hassoun PM, et al. Sphingosine 1-phosphate reduces vascular leak in murine and canine models of acute lung injury. Am J Respir Crit Care Med 2004;170:987-993.

2. Horgan MJ, Fenton JW, Malik AB. Alpha-thrombininduced pulmonary vasoconstriction. J Appl Physiol 1987;63:1993-2000.

3. Garcia JG, Slflinger-Birnbolm A, Bizlos R, et al. Thrombininduced increase in albumin permeability across the endothelium. J Cell Physiol 1986;128:96-104.

4. Dubrovskyi O, Birukova AA, Birukov KG. Measurement of local permeability at subcellular level in cell models of 
agonist- and ventilator-induced lung injury. Lab Invest 2013;93:254-263 (this issue).

5. Hubmayr R, Plataki M. The Physical Basis of VentilatorInduced Lung Injury. Expert Rev Respir Med 2010;4:373-385.

6. Birukova AA, Chatchavalvanich $\mathrm{S}$, Rios $\mathrm{A}$, et al Differential regulation of pulmonary endothelial monolayer integrity by varying degrees of cyclic stretch. Am J Pathol 2006;168:1749-1761.

7. Dudek SM, Garcia JGN. Cytoskeletal regulation of pulmonary vascular permeability. J Appl Physio 2001;91:1487-1500.

8. Krishnan R, Klumpers DD, Park $C Y$, et al. Substrate stiffening promotes endothelial monolayer disruption through enhanced physical forces. Am J Physiol Cell Physiol 2011;300:C146-C154.

9. Dudek SM, Garcia JG. Cytoskeletal regulation of pulmonary vascular permeability. J Appl Physiol 2001:91:1487-1500.
10. Dejana E. Endothelial cell-cell junctions: happy together. Nat Rev Mol Cell Biol 2004;5:261-270.

11. Butler JP, Tolic-Norrelykke IM, Fabry B et al. Traction fields, moments, and strain energy that cells exert on their surroundings. Am J Physiol Cell Physiol 2002;282:C595-C605.

12. Tambe DT, Hardin C, Angelini TE, et al. Collective cell guidance by cooperative intercellular forces. Nat Mater 2011;10:469-475.

13. Trepat $\mathrm{X}$, Wasserman MR, Angelini TE, et al. Physical forces during collective cell migration. Nat Phys 2009;5:426-430.

14. Angelini $T E$, Hannezo $E$, Trepat $X$, et al. Glass-like dynamics of collective cell migration. Proc Natl Acad Sci USA 2011;108:4714-4719.

15. Angelini TE, Hannezo E, Trepat $X$, et al. Cell migration driven by cooperative substrate deformation patterns. Phys Rev Lett 2010;104:168104. 